\title{
TI.5.1
}

\section{Final Report of the Per-Entity Metadata Working Group}

- PDF: TI.5.1-FinalReportofthePer-EntityMetadataWorkingGroup.pdf

- Text: TI.5.1-FinalReportofthePer-EntityMetadataWorkingGroup.txt

\section{More Information}

\begin{tabular}{|l|l|}
\hline Repository ID & TI.5.1 \\
\hline Persistent URL & http://doi.org/10.26869/TI.5.1 \\
\hline Title & Final Report of the Per-Entity Metadata Working Group \\
\hline Authors & Scott Koranda, David Walker, The Per-Entity Metadata Working Group \\
\hline Sponsor & InCommon TAC \\
\hline Review & Consultation for the Per-Entity Metadata Working Group Final Report \\
\hline Status & Preserve \\
\hline Publish Date & $12 / 7 / 2016$ \\
\hline DOI & $10.26869 /$ TI.5.1 \\
\hline Signature & \\
\hline Deprecated & No \\
\hline Future Review & $12 / 1 / 2017$ \\
\hline Supersedes & \\
\hline Format & PDF \\
\hline Related Docs & \\
\hline Development Location & Per-Entity Metadata Working Group \\
\hline IP Framework & CC BY 4.0 \\
\hline Subject Tags & \\
\hline Notes & \\
\hline
\end{tabular}

